\title{
Medikationssicherheit: Wo steht die Schweiz?
}

Englischer Titel: Medication Safety in Switzerland: Where are we today?

\section{Autoren und Affiliationen}

Dr. med. Liat Fishman ${ }^{1}$, Dr. Lea Brühwiler, Prof. Dr. David Schwappach, MPH ${ }^{1,2}$

${ }^{1}$ Stiftung Patientensicherheit Schweiz. Zürich, Schweiz

${ }^{2}$ Institut für Sozial- und Präventivmedizin (ISPM), Universität Bern. Bern, Schweiz.

Korrespondenzadresse: David Schwappach, Patientensicherheit Schweiz, Asylstrasse 77, 8032

Zürich, Schweiz, Tel. +41(0)43 24414 80, Fax +41(0)43 2441481 ,

schwappach@patientensicherheit.ch

Zeichenzahl des Textes ohne Zusammenfassungen und Literaturverzeichnis: 20‘883

Zeichenzahl der deutschen Zusammenfassung: 1645

Zeichenzahl der englischen Zusammenfassung: 1538

Zahl der Referenzen: 44

Zahl der Abbildungen: 1 


\section{Zusammenfassung Deutsch}

Empirische Analysen zeigen, dass die Medikationssicherheit ein aktueller und drängender Problembereich der Schweizer Gesundheitsversorgung ist. Unerwünschte Arzneimittelereignisse und Medikationsfehler kommen häufig vor und Risiken wie Polypharmazie sind weit verbreitet. Es gibt in der Schweiz keine umfassende nationale Strategie, die sich explizit der Medikationssicherheit widmet. Der Föderalismus mit relativer Autonomie der Kantone bei den Gesetzen der Gesundheitsversorgung beeinflusst die Umsetzung nationaler Reformen im Gesundheitswesen, auch zum Nachteil der Arzneimitteltherapiesicherheit. Eine Besonderheit der Schweiz ist die ärztliche Medikamentenabgabe, die in fast allen Deutschschweizer Kantonen erlaubt ist und spezifische Herausforderungen für die Medikationssicherheit impliziert. Gleichwohl existieren zunehmend Aktivitäten auf nationaler Ebene, die verschiedene Aspekte der Medikationssicherheit behandeln, wie die „progress!“-Programme im Rahmen der nationalen Qualitätsstrategie. Im Nationalen Forschungsprogramm "Gesundheitsversorgung“ (NFP 74) des Schweizer Nationalfonds bearbeiten mehrere Forschungsprojekte aktuell die Medikationssicherheit. Klinisch-pharmazeutische Aktivitäten in Krankenhäusern sind verhältnismäßig weit verbreitet. Pharmaceutical Care in der Grundversorgung und die entsprechenden Kompetenzen für Apotheker werden ausgebaut. Dennoch braucht es eine gesamtheitliche Strategie, Prioritätensetzung und Wirksamkeitsprüfung unter Einbindung aller Stakeholder damit das Schweizer Gesundheitswesen die Herausforderungen, die sich für die Medikationssicherheit stellen, zukunftsgerichtet begegnen kann.

Schlüsselwörter: Medikationssicherheit, Arzneimitteltherapiesicherheit, Versorgungsqualität, Versorgungskoordination, Schweiz

\section{Abstract English}

Empirical research shows that medication safety is an urgent area of concern in the Swiss health care system. Adverse drug events and medication errors are common and risks such as polypharmacy are widespread. No comprehensive national strategy explicitly dedicated to medication safety exists in Switzerland. The federalist system of government with relative autonomy of the cantons relating to health care laws influences the implementation of national health care reforms, also to the disadvantage of medication safety. Direct dispensing of drugs by the prescribing physician is permitted in almost all German-speaking cantons. This special feature of the Swiss system implies specific challenges for medication safety. Nonetheless, there is an increasing number of national activities dealing with various aspects of medication safety, such as the "progress!" programmes within the National Quality Strategy. Within the National Research Programme "Smarter Health Care" (NRP 74) of the Swiss National Science Foundation several research projects are currently focusing on medication safety. Clinical pharmacy activities in hospitals are relatively widespread. In the primary care sector, pharmaceutical care practice and the corresponding competencies for pharmacists are 
being further developed. However, a comprehensive strategy, priority-setting and effectiveness studies involving all stakeholders are required in order for the Swiss health care system to meet the challenges facing medication safety in a forward-looking manner.

Keywords: medication safety, quality of care, care coordination, public health, Switzerland 


\section{Einleitung}

Probleme in der Medikationssicherheit stehen auch in der Schweiz zunehmend im fachlichen und öffentlichen Interesse. Im folgenden Beitrag geben wir einen Überblick zum Status quo der Arzneimitteltherapiesicherheit (AMTS ${ }^{1}$ ) in der Schweiz. Um die Ausgangslage zu illustrieren, werden zunächst Daten zur Medikationssicherheit und zum Auftreten von unerwünschten Arzneimittelereignissen (UAE) vorgestellt. Anschliessend gehen wir auf besondere Rahmenbedingungen des Schweizer Gesundheitssystems ein, die sich auf die Medikationssicherheit auswirken. Im zweiten Teil stellen wir Initiativen und Aktivitäten zur Medikationssicherheit in der Schweiz vor.

\section{Ausgangslage}

\section{Epidemiologie}

Auf nationaler Ebene durchgeführte Studien zum Auftreten von UAE und Medikationsfehlern fehlen in der Schweiz. Es gibt jedoch einige, allerdings häufig ältere, Untersuchungen, welche Hinweise zum Ausmass der Problematik geben - und aufzeigen, dass die Medikationssicherheit einen Hotspot der Patientensicherheit in der Schweiz darstellt.

Schweizer Daten zum Auftreten von UAE in Krankenhäusern spiegeln in etwa die internationalen Raten wider. In einem von 1974 bis 1993 laufenden Monitoring-Programm wurden bei 48.005 konsekutiven Hospitalisationen 12.785 (27\%) unerwünschte Arzneimittelwirkungen (UAW) erfasst [1]. Spätere Studien ermittelten, dass $8-15 \%$ der stationären Patienten ${ }^{2}$ von einem UAE betroffen sind [24] und 4-7\% der Krankenhausaufnahmen auf UAE zurückzuführen sind [3, 5, 6]. In einer Aktenanalyse in einem Krankenhaus machten UAE die Hälfte der unerwünschten Ereignisse bei internmedizinischen Hospitalisationen aus [7]. In einem weiteren Krankenhaus zeigte eine Analyse der im Fehlermeldesystem berichteten Ereignisse, dass Medikationsfehler sich am häufigsten als Fehlerkette in den Phasen der Vorbereitung bis zur Abgabe eines Medikaments (45\% der Fehler) ereigneten [8]. Auch illustrieren Untersuchungen zu Medikationsdiskrepanzen bei Krankenhausaufnahme und entlassung, dass diese Behandlungsübergänge im Schweizer Gesundheitssystem ernstzunehmende Risiken für Patienten darstellen. So wurde in einer Studie in einer kardiologischen Universitätsklinik festgestellt, dass $9 \%$ der von Patienten zuhause eingenommenen Medikamente weder im

\footnotetext{
${ }^{1}$ Die Begriffe AMTS und Medikationssicherheit werden synonym verwendet.

${ }^{2}$ Wir verwenden in diesem Beitrag manchmal männliche, manchmal weibliche Bezeichnungen.

Selbstverständlich sind immer beide Geschlechter eingeschlossen.
} 
Überweisungsbrief noch auf persönlichen Medikationslisten der Patienten aufgeführt waren [9]. Eine andere Untersuchung zeigte, dass in internistischen Entlassungsbriefen sehr häufig Medikamente ohne Indikation verordnet wurden (55\% der Patienten) und Medikamente ausgelassen wurden (44\%) [10]. Auch eine in Offizinapotheken durchgeführte Studie fand bei 55\% der Entlassrezepte von Krankenhäusern Unklarheiten, Verordnungsfehler oder andere arzneimittel-bezogene Probleme [11].

Die wenigen vorhandenen Daten aus dem ambulanten Sektor deuten darauf hin, dass auch hier Handlungsbedarf besteht. Eine Befragung beim Personal in Arztpraxen zeigte, dass die Medikationssicherheit als prioritäres Handlungsfeld eingeschätzt wird [12, 13]. Eine bei Hausärzten und Pädiatern durchgeführte prospektive Studie schlussfolgerte, dass UAE bei Erwachsenen häufiger als in der Pädiatrie vorkommen (46,5 pro 100.000 Patientenkontakte vs. 2,8 pro 100.000 Patientenkontakte). Ältere und multimorbide Patienten waren besonders gefährdet und oft spielten Kommunikationsprobleme eine Rolle [14].

Auch aus Sicht der Schweizer Bevölkerung sind Medikationsfehler häufig. In der repräsentativen International Health Policy Survey Studie 2010 gaben 5,3\% der befragten Schweizer an, innerhalb der letzten zwei Jahre einen Medikationsfehler erlebt zu haben [15]. Im International Health Policy Survey 2017 berichtete knapp die Hälfte der über 65-jährigen Schweizer mit mindestens zwei verordneten Medikamenten, dass innen im letzten Jahr die Nebenwirkungen ihrer Arzneimittel erklärt und eine Medikamentenliste ausgehändigt wurde [16]. Hinsichtlich dem Patienteneinbezug bei der Therapie besteht also deutliches Optimierungspotenzial. Sprachbarrieren, die durch den hohen Anteil an Migrationsbevölkerung häufig vorkommen, gefährden die Medikationssicherheit zusätzlich, wie eine Befragung von Apothekern deutlich zeigte [17].

Zudem ist in der Schweiz die Polypharmazie weit verbreitet. Aktuelle Studien zeigen, dass $18-25 \%$ der über 65-jährigen Bevölkerung mindestens fünf Medikamente regelmässig einnehmen [16, 18]. Eine Analyse der Bezugsdaten der größten Schweizer Krankenkasse Helsana wies eine noch höhere Prävalenz von 50\% auf, wobei die unterschiedliche Methodik zu berücksichtigen ist. Im Schnitt bezog die über 65-jährige Bevölkerung parallel 5,6 Medikamente [19]. Erstmals wurden auch die Medikamentenbezüge bei Pflegeheimbewohnern gesondert ausgewertet. Es zeigte sich, dass diese im Zeitraum 2013-2016 stetig stiegen. Pflegeheimbewohner über 65 Jahre bezogen durchschnittlich 9,3 Medikamente gleichzeitig. Fast vier von fünf Bewohnern bezogen in 2016 mindestens ein potenziell inadäquates Medikament (PIM) ${ }^{3}$ [19]. Die Daten bestätigen, dass die vulnerable Gruppe der Pflegeheimbewohner bei Aktivitäten zur Verbesserung der AMTS besonders im Fokus stehen sollte.

${ }^{3}$ Zur Identifizierung der einzelnen PIM dienten die Beers Criteria sowie die Priscus-Liste [20, 21]. 


\section{Besondere Rahmenbedingungen}

\section{$\underline{26 \text { Kantone, } 26 \text { Gesundheitssysteme }}$}

Der Föderalismus mit der Aufteilung von Zuständigkeiten zwischen Bund ${ }^{4}$, Kantonen und Gemeinden ist ein prägendes Merkmal des Schweizer Gesundheitssystems. So ist der Bund zum Beispiel verantwortlich für die obligatorische Krankenversicherung und die Bekämpfung übertragbarer Krankheiten. Demgegenüber regeln die Kantone weitgehend autonom die Gesundheitsversorgung, beispielsweise die Krankenhausversorgung. Die Aufgaben der Gemeinden umfassen etwa die Organisation der häuslichen Pflege. Da das Krankenversicherungsgesetz wichtige gesundheitspolitische Fragen auf nationaler Ebene regelt, gibt es teilweise überlappende, aber oft unzulänglich koordinierte Zuständigkeiten.

Die gesetzlichen Vorgaben zur Gesundheitsversorgung variieren teilweise erheblich von Kanton zu Kanton. Eines von vielen Beispielen ist die heterogene Regelung betreffend der ärztlichen Versorgung von Pflegeheimen - das Spektrum reicht dabei von der obligatorischen Mitgliedschaft einer Ärztin in der Heimleitung bis zum Fehlen jeder Vorgabe [22]. Auch die Aufgaben der Kantonsärzte und apotheker, die in jedem Kanton für die Umsetzung der Gesetze verantwortlich sind, unterscheiden sich zwischen den Kantonen.

Der Föderalismus erklärt zum Teil die Schwierigkeit beim Einleiten nationaler Reformen. Die Gesellschaft ist bei staatlichen Interventionen und Regulierung generell zurückhaltend, sodass Innovationen in der Gesundheitsversorgung eher über „peer pressure“ und Bottom-up-Ansätze erfolgen und in der Umsetzung tendenziell langsam sind.

Ein Beispiel für eine nationale Reform, die sich immer wieder verzögert hat und bei der die Schweiz anderen Ländern deutlich hinterherhinkt [23], ist die Einführung intersektoraler elektronischer Patientenakten. Hier stellt nicht nur die beschriebene institutionelle Fragmentierung im Gesundheitswesen eine zentrale Barriere dar, sondern auch die Fragmentierung auf organisationaler Ebene, die sich durch die Vielzahl der Gesundheitsdienstleister und die strikte Trennung zwischen den Krankenkassen und Leistungserbringern äussert [24]. Im Jahr 2017 ist schliesslich das Bundesgesetz über das elektronische Patientendossier (EPDG) in Kraft getreten (www.bag.admin.ch). Es gibt vor, dass Krankenhäuser bis 2020 und Pflegeheime bis 2022 ein elektronisches Patientendossier (EPD) anbieten müssen. Für ambulante Arztpraxen ist die Teilnahme freiwillig - was eine wesentliche Barriere für eine erfolgreiche Verbreitung sein kann. Das EPD ist dabei kein einheitliches Dossier für die ganze Schweiz, sondern ein dezentraler Zusammenschluss von einzelnen autonomen Projekten,

\footnotetext{
${ }^{4}$ Der „Bund“ entspricht dem Staat Schweiz mit den drei Gewalten Bundesrat (Exekutive), Parlament (Legislative) und Bundesgerichtshof (Judikative).
} 
in denen Gesundheitsfachpersonen und ihre Institutionen sich jeweils in einer "Gemeinschaft" organisieren. Unter diesen Bedingungen ist das Schweizer EPD insgesamt ein sehr anspruchsvolles Vorhaben mit extrem hohem Koordinationsaufwand.

Die eMedikation ist ein zentraler Bestandteil des EPD. Bereits seit einigen Jahren gibt es EPDkompatible Pilotprojekte in einzelnen Kantonen bei denen Medikationsdaten zwischen Leistungserbringern ausgetauscht werden - zum Beispiel in Genf und im Kanton Waadt [25]. Erwähnenswert ist auch der eMediplan, der zunächst in den Kantonen Thurgau, St. Gallen und Zug implementiert wird. Wie in Deutschland handelt es sich dabei um einen Medikationsplan, der mittels 2D-Barcode auf Papier übertragen werden kann - er erfüllt aber auch die Voraussetzungen für einen elektronischen Datenaustausch im EPD [26]. Auch bei diesem Projekt ist die Umsetzung langsamer als geplant, dürfte aber durch das Inkrafttreten des EPDG an Auftrieb gewinnen.

Nicht nur institutionsübergreifend, auch innerhalb von Institutionen sind die genutzten elektronischen Systeme häufig nicht miteinander kompatibel, was eine Gefahr für die Medikationssicherheit darstellen kann. Generell ist der Implementierungsgrad elektronischer Patientenakten und Verordnungssysteme in Schweizer Krankenhäusern variabel. Mehrere Universitätskrankenhäuser haben elektronische Verordnungssysteme für ihre eigenen Bedürfnisse weiterentwickelt.

\section{$\underline{\text { Ärztliche Medikamentenabgabe (Selbstdispensation) }}$}

Derzeit dürfen die Ärzte in 15 Kantonen in der Deutschschweiz Medikamente direkt an ihre Patienten verkaufen und abgeben, in neun Kantonen ist die Selbstdispensation verboten und in zwei Kantonen werden Mischsysteme angewandt. Befürworter der Selbstdispensation argumentieren, dass diese für eine schnelle und breite Versorgung mit Medikamenten sorgt [27]. Für den Medikationsprozess bedeutet dies allerdings, dass die Rezeptvalidierung durch die abgebende Apotheke entfällt, was das Risiko für arzneimittelbezogene Probleme erhöhen kann. Eine Analyse der Helsana-Krankenkasse zeigte, dass Personen welche die Mehrheit ihrer Medikamente von ihrem Arzt erhielten, eine 15\% grössere Wahrscheinlichkeit hatten ein PIM zu erhalten, als wenn die verschriebenen Medikamente in der Apotheke bezogen wurden [28].

Zudem wirkt sich der Wettbewerb zwischen Ärzten und Apothekern hemmend auf die interprofessionelle Zusammenarbeit aus. Beispielsweise haben sich in den letzten 20 Jahren in den Westschweizer (mehrheitlich französischsprachigen) Kantonen - in denen die Selbstdispensation nicht erlaubt ist - Qualitätszirkel mit Ärzten und Apothekern etabliert, während diese in den deutschschweizer Kantonen bislang wenig verbreitet sind. Eine im Kanton Freiburg durchgeführte Analyse zeigte deutlich geringere Verschreibungskosten der in Qualitätszirkeln involvierten Ärzte, unter anderem aufgrund eines reflektierteren Umgangs mit Marketingstrategien der Medikamentenhersteller [29]. Bislang existiert aber noch wenig Evidenz zu den Auswirkungen der Qualitätszirkel auf die Medikationssicherheit. 
Analog ist in der Westschweiz die pharmazeutische Betreuung von Pflegeheimen intensiver als in der Deutschschweiz. In den Kantonen Waadt, Wallis und Freiburg analysieren die zuständigen Apothekerinnen den Medikamentenverbrauch und formulieren Empfehlungen, die als Grundlage für Qualitätszirkel mit der Pflege, Ärzte- und Apothekerschaft dienen [30]. Die Konkurrenz bei der Medikamentenabgabe hat auch Auswirkungen auf die Zusammenarbeit der Berufsgruppen im stationären Sektor: So zeigten Messerli et al. eine deutliche Assoziation zwischen der Selbstdispensation im ambulanten Sektor und der Abwesenheit klinisch-pharmazeutischer Dienstleistungen in den Krankenhäusern (Abbildung 1) [31].

\section{Aktivitäten mit Bezug zur Medikationssicherheit}

Hier werden vor allem zentrale Strategien und Aktivitäten von nationaler Tragweite vorgestellt. Aus Platzgründen erhebt die Darstellung keinen Anspruch auf Vollständigkeit. Es existieren auf den unterschiedlichsten Ebenen und bei den unterschiedlichsten Leistungserbringern weitere Aktivitäten.

\section{Allgemeine nationale Strategien und Aktivitäten}

In der Schweiz gibt es keine umfassende nationale Strategie die sich explizit der AMTS widmet, die also etwa vergleichbar wäre mit dem deutschen Aktionsplan des Bundesministeriums für Gesundheit. Zwar ist eines der Ziele der umfangreichen Strategie Gesundheit2020 des Bundes aus dem Jahr 2013 die Erhöhung der Versorgungsqualität, dennoch ist die Medikationssicherheit kein explizit genanntes Handlungsfeld. Es gibt keine nationalen, staatlich mandatierten Institute, die sich mit Qualitäts- und Sicherheitsfragen beschäftigen, wie das Institut für Qualität und Wirtschaftlichkeit im Gesundheitswesen (IQWIG) oder das Institut für Qualitätssicherung und Transparenz im Gesundheitswesen (IQTIG). Entsprechende Überlegungen wurden durch viele Stakeholder abgelehnt. Dies führt unter anderem dazu, dass es sehr wenige qualitätsbezogene Daten gibt, insbesondere für den ambulanten Bereich, und dass eine nationale Steuerung und konzertierte Aktivitäten nur bedingt existieren. Gleichwohl behandeln einige nationale Aktivitäten im Bereich Qualität der Gesundheitsversorgung die Medikationssicherheit.

\section{Nationale Qualitätsstrategie des Bundes}

Um die Qualität der medizinischen Leistungen weiterzuentwickeln verabschiedete der Bund 2009 eine nationale Qualitätsstrategie [32]. Schwerpunkte sind die bessere Koordinierung der Qualitätssicherung, der Ausbau von Health Technology Assessment (HTA) und von Qualitätsindikatoren insbesondere für den ambulanten Bereich sowie die Durchführung nationaler Verbesserungsprogramme in zentralen Bereichen der Patientensicherheit. 
Bislang wurden unter maßgeblicher Finanzierung des Bundesamts für Gesundheit (BAG) vier solche Verbesserungsprogramme unter dem Namen „progress!“ initiiert. Verantwortlich für die Entwicklung und Umsetzung ist die Stiftung Patientensicherheit Schweiz. Das Programm progress! Sichere Medikation an Schnittstellen (2014-2017) fokussierte auf die Implementierung des systematischen Medikationsabgleichs (Medication Reconciliation) in Krankenhäusern. Es bestand zum einen aus einer Kampagne zur Sensibilisierung von Fachwelt und Öffentlichkeit. Parallel wurde ein Vertiefungsprojekt mit acht Pilotkrankenhäusern durchgeführt, um Erfahrungen mit dem Medikationsabgleich bei Krankenhausaufnahme zu sammeln [33]. Zum Abschluss des Programms veröffentlichte Patientensicherheit Schweiz zusammen mit zentralen Stakeholdern im Gesundheitswesen eine Charta, die den Medikationsabgleich zu einem notwendigen Standard erklärt und wichtige Rahmenbedingungen für die Umsetzung beschreibt [34].

Zentrales Thema im jüngsten Programm progress! Sichere Medikation in Pflegeheimen ist die Reduktion von Polypharmazie sowie ein adäquater Umgang mit PIM bei Bewohnenden von Pflegeheimem [35]. In den Jahren 2017 und 2018 werden zunächst Grundlagen erarbeitet. Aufgrund der komplexen heterogenen Ausgangslage (Föderalismus, Organisation der Heime) soll erst in einer zweiten Phase ab 2019 ein Vertiefungsprojekt durchgeführt werden, in dem ausgewählte Verbesserungsmaßnahmen auf ihre Umsetzbarkeit und Wirksamkeit untersucht werden.

\section{Stiftung Patientensicherheit Schweiz}

Patientensicherheit Schweiz ist das nationale Kompetenzzentrum für die Entwicklung und Förderung der Patientensicherheit im schweizerischen Gesundheitswesen (www.patientensicherheit.ch). Im Rahmen von Forschungsprojekten, nationalen Programmen sowie Bildungsangeboten befasst sich die Stiftung mit verschiedenen Aspekten der Patientensicherheit. Neben den bereits genannten progress!-Programmen werden weitere Themen der Medikationssicherheit bearbeitet. Aktuell werden beispielsweise basierend auf Forschungsarbeiten Empfehlungen zur (Doppel-)kontrolle von Hochrisiko-Medikationen entwickelt [36-38]. Zudem werden aus der CIRRNET-Datenbank, einem Netzwerk lokaler Fehlermeldeysteme (CIRS), unter anderem überregionale Probleme der Medikationssicherheit identifiziert und in knapp gefassten Verbesserungsempfehlungen, den QuickAlerts, bearbeitet. Die Stiftung wird vom Bund, den Kantonen und wichtigen Stakeholdern getragen. Die Finanzierung erfolgt zu zwei Dritteln auf Projektbasis, wobei angestrebt wird, eine langfristige Grundfinanzierung zu erreichen, da unter den derzeitigen Rahmenbedingungen die Etablierung einer nachhaltigen Strategie erschwert ist.

\section{Nationales Forschungsprogramm „Gesundheitsversorgung" (NFP 74)}

Der Bundesrat definiert die Themen der nationalen Forschungsprogramme. Mit dem NFP 74 wurde erstmals die Versorgungsforschung als Themenbereich ausgewählt (www.nfp74.ch). Dies ist insofern 
bedeutsam, als die Versorgungsforschung in der Schweiz mit Ausnahme weniger „Inseln“ bislang wenig etabliert ist. Vier Forschungsprojekte des NFP 74 befassen sich seit 2017 gezielt mit der Medikationssicherheit: „Automatische Erkennung medikamentöser Nebenwirkungen in der Geriatrie“, „Optimierte Medikation und Kommunikation bei Spitalaustritt“, „Optimierung der Medikation in Altersund Pflegeheimen“ sowie „Verbesserte Medikation bei chronisch Kranken dank elektronischen Entscheidungshilfen“.

\section{Nationale Datenbank zur Dosierung von Arzneimitteln bei Kindern}

Im Jahr 2008 begann der Pharmazeutische Dienst des Kinderspitals Zürich mit der Entwicklung einer Kinderdosierungsdatenbank; 2012 ging die Website www.kinderdosierungen.ch online. In 2017 gründeten die acht größten Kinderkliniken der Schweiz zusammen mit der Schweizerischen Gesellschaft für Pädiatrie und dem Schweizerischen Verein für Amts- und Spitalapotheker (GSASA) den Verein SwissPedDose (www.swisspeddose.ch). Das BAG beauftragte den Verein, ab 2018 eine nationale Datenbank mit Empfehlungen zur Arzneimitteldosierung in der Pädiatrie zu betreiben. Die Dosierungsempfehlungen werden laufend in einem standardisierten Konsensverfahren entwickelt und stehen allen Gesundheitsfachpersonen zur Verfügung.

\section{Pharmakovigilanz-Meldesystem}

Das Schweizerische Heilmittelinstitut Swissmedic (www.swissmedic.ch) hat die Funktion des nationalen Pharmakovigilanz-Zentrums inne. Es empfängt einerseits UAW-Meldungen von sechs regionalen Zentren, welche einer universitären Abteilung für klinische Pharmakologie angegliedert sind. Die regionalen Zentren evaluieren und bearbeiten UAW-Meldungen von Fachpersonen oder Konsumentinnen und leiten sie an Swissmedic weiter. Die pharmazeutische Industrie sendet ihrerseits an Swissmedic UAW-Meldungen weiter, die bei ihr eingegangen sind. Swissmedic leitet bei Bedarf mit den für das Präparat zuständigen Abteilungen Massnahmen ein. Ein Beispiel dafür ist die Erstellung eines Massnahmenplans in Zusammenarbeit mit den Herstellerfirmen aufgrund sich wiederholender Berichte über schwerwiegende Komplikationen durch tägliche statt wöchentlicher Verabreichung von Low-Dose Methotrexat bei Patienten mit rheumatoider Arthritis oder Psoriasis [39]. Obwohl das Heilmittelgesetz die UAW-Meldepflicht für medizinische Fachpersonen vorsieht, ist von einem ausgeprägten Underreporting auszugehen [40]. Um hier Abhilfe zu schaffen, wurde von einer Arbeitsgruppe ein elektronisches System entwickelt, das eine aktive Überwachung der UAW bei hospitalisierten Patienten erlaubt [40].

\section{Klinisch-pharmazeutische Aktivitäten}


Die Krankenhausapotheker in der Schweiz sind in der GSASA organisiert und engagieren sich vielseitig für die Medikationssicherheit. In größeren Krankenhäusern ist die klinische Pharmazie verhältnismäßig weit verbreitet, auch wenn es regionale Unterschiede gibt. In der französischen Westund italienischen Süd-Schweiz wurden bereits früh klinische Aktivitäten initiiert. Dort machten die klinischen Pharmazeuten in 2013 in größeren Spitälern einen Viertel der Apothekerstellen aus, während dieser Anteil in der Deutschschweiz nur knapp 10\% betrug (Abbildung 1) [31]. Die Ressourcen werden vor allem für die Visitenbegleitung eingesetzt, wogegen Aktivitäten zur Gewährleistung der Versorgungskontinuität (Seamless care) noch wenig etabliert sind. Kleine Krankenhäuser haben oft nur wenig akademisch-pharmazeutische Beratung. Zunehmend verbreitet sich deshalb die Einbindung von Pharmaassistentinnen (entspricht in Deutschland den pharmazeutisch-technischen Assistentinnen), zum Beispiel in der Stationsbewirtschaftung oder dem patientenindividuellen Richten der Medikamente. In progress! Sichere Medikation an Schnittstellen wurden ausserdem erste Ansätze zur Einbindung von Pharmaassistentinnen beim Medikationsabgleich erprobt [41].

Den Schweizer Apothekern kommt auch in der Grundversorgung eine wachsende Rolle zu [42]. In einer europaweiten Befragung von Offizinapothekern in 2012 schnitt die Schweiz in Bezug auf routinemässige Aktivitäten der pharmazeutischen Betreuung (Pharmaceutical care) insgesamt am Besten ab [43]. Schweizer Apothekerinnen führten überdurchschnittlich häufig Aktivitäten in der direkten Patientenversorgung durch, zum Beispiel das Sicherstellen, dass der Patient gegebene Informationen versteht. Seit 2010 können Offizinapotheker in der Schweiz jenen Patienten mit einer Langzeitanwendung von mindestens vier Medikamenten auf Kosten der Krankenkasse einen „Medication review“, den Polymedikations-Check, anbieten [44]. Daneben fördert der Apothekerverband pharmaSuisse zunehmend auch die Entwicklung weiterer Dienstleistungen wie die telemedizinisch unterstützte Beratungsmöglichkeit „netCare“ [42].

\section{Fazit und Ausblick}

In der Schweiz existieren zur Medikationssicherheit viele wertvolle Einzelaktivitäten der verschiedensten Akteure, die hier nicht vollständig aufgeführt wurden. Was jedoch fehlt, sind eine gesamtheitliche Strategie, Prioritätensetzung und Wirksamkeitsprüfung unter Einbindung und Vernetzung aller Stakeholder - sowie Institutionen, die eine solche Strategie nachhaltig etablieren könnten. Die Versorgungsforschung, die Etablierung der klinischen Pharmazie und das Empowerment der Bevölkerung müssen mehr gefördert werden. Auch müssen existierende Strukturen und Akteure gestärkt und ihre Arbeit finanziell langfristig abgesichert werden. Der ambulante Bereich sollte wie in den meisten Ländern stärker in den Fokus rücken und dort eine valide Datenbasis geschaffen werden. Eine kontinuierliche Aufgabe des gesamten Gesundheitswesens ist ferner die Sensibilisierung der Fachpersonen für die Risiken im Medikationsprozess. Dazu gehört auch die Verankerung der Patienten- und Medikationssicherheit in der Primärausbildung. 
Themen mit hoher Aktualität im Schweizer Gesundheitswesen sind neue Modelle der interprofessionellen Zusammenarbeit und die Digitalisierung mit Einführung des EPD. Auch wenn der Weg noch lang ist, können diese Entwicklungen als wichtige Treiber für die Medikationssicherheit gesehen werden.

Seit etwa fünf Jahren existieren Bestrebungen seitens des Bundes, Qualitätsaktivitäten national zu konzentrieren, um eine übergeordnete Koordination und Überwachung der Qualität im Gesundheitswesen zu ermöglichen. Der aktuelle Vorschlag strebt einen langfristigen und stabilen Finanzierungsmechanismus für nationale Qualitäts- und Patientensicherheitsaktivitäten an, der von allen Stakeholdern unterstützt wird.

Die Vielfalt an Akteuren und die wachsende Zahl chronisch kranker, multimorbider Patientinnen und Patienten stellen grosse Herausforderungen für die Medikationssicherheit dar. Auch die Schweiz mit ihrem hochqualitativen Gesundheitssystem muss diesem Thema aktiver begegnen und braucht eine kohärente Strategie um bei den Herausforderungen zukunftsgerichtet Fortschritte zu erzielen.

\section{Danksagung}

Wir danken Dr. Markus Messerli, Universität Basel für die Überlassung der Abbildung.

\section{Interessenkonflikte}

Die Autoren arbeiten für Patientensicherheit Schweiz und bekunden keine weiteren Interessenkonflikte. 


\section{Literaturverzeichnis}

1. Hunziker T, Bruppacher R, Kuenzi U P, et al. (2002) Classification of ADRs: a proposal for harmonization and differentiation based on the experience of the Comprehensive Hospital Drug Monitoring Bern/St. Gallen, 1974-1993. Pharmacoepidemiol Drug Saf 11: 159-63

2. Fattinger K, Roos M, Vergeres $\mathrm{P}$, et al. (2000) Epidemiology of drug exposure and adverse drug reactions in two swiss departments of internal medicine. Br Clin Pharmacol 49: 158-67

3. Hardmeier B, Braunschweig S, Cavallaro M, et al. (2004) Adverse drug events caused by medication errors in medical inpatients. Swiss Med Wkly 134: 664-70

4. Schlienger R G, Luscher T F, Schoenenberger R A, Haefeli W E (1999) Academic detailing improves identification and reporting of adverse drug events. Pharm World Sc 21: 110-5

5. Wasserfallen J, Livio F, Buclin T, Tillet L, Yersin B, Biollaz J (2001) Rate, type, and cost of adverse drug reactions in emergency department admissions. Eur J Intern Med 12: 442-7

6. Lepori V, Perren A, Marone C (1999) Unerwünschte internmedizinische Arzneimittelwirkungen bei Spitaleintritt [Adverse internal medicine drug effects at hospital admission]. Schweiz Med Wochenschr 129: 915-22

7. Halfon P, Staines A, Burnand B (2017) Adverse events related to hospital care: a retrospective medical records review in a Swiss hospital. Int J Qual Health Care 29: 527-33

8. Huckels-Baumgart S, Manser T (2014) Identifying medication error chains from critical incident reports: A new analytic approach. The Journal of Clinical Pharmacology 54: 1188-97

9. Frei P, Huber L C, Simon R W, Bonani M, Luscher T F (2009) Insufficient medication documentation at hospital admission of cardiac patients: a challenge for medication reconciliation. J Cardiovasc Pharmacol 54: 497-501

10. Perren A, Previsdomini M, Cerutti B, Soldini D, Donghi D, Marone C (2009) Omitted and unjustified medications in the discharge summary. Qual Saf Health Care 18: 205-8

11. Eichenberger P M, Lampert M L, Kahmann I V, van Mil J W, Hersberger K E (2010) Classification of drug-related problems with new prescriptions using a modified PCNE classification system. Pharm World Sc 32: 362-72

12. Gehring K, Schwappach D L B, Battaglia M, et al. (2012) Frequency of and Harm Associated With Primary Care Safety Incidents. Am J Manag Care 18: e323-e37

13. Schwappach D L B, Gehring K, Battaglia M, et al. (2012) Threats to patient safety in the primary care office: concerns of physicians and nurses. Swiss Med Wkly 142: w13601

14. Gnädinger M, Conen D, Herzig L, et al. (2017) Medication incidents in primary care medicine: a prospective study in the Swiss Sentinel Surveillance Network. BMJ Open 7: e013658

15. Schwappach D L B (2012) Risk factors for patient-reported medical errors in eleven countries. Health Expect 17: 321-31

16. Merçay $C$ (2017) Expérience de la population âgée de 65 ans et plus avec le système de santé. Analyse de l'International Health Policy Survey 2017 de la fondation Commonwealth Fund sur mandat de l'Office fédéral de la santé publique (OFSP) (Obsan Dossier 60). https://www.obsan.admin.ch/sites/default/files/publications/2017/obsan dossier 60 1.pdf Zugegriffen: 22. Feb. 2018

17. Schwappach D L, Meyer Massetti C, Gehring K (2012) Communication barriers in counselling foreign-language patients in public pharmacies: threats to patient safety? Int J Clin Pharm 34: 765-72

18. Castioni J, Marques-Vidal P, Abolhassani N, Vollenweider P, Waeber G (2017) Prevalence and determinants of polypharmacy in Switzerland: data from the CoLaus study. BMC Health Serv Res 17: 840

19. Schneider R, Schur N, Reinau D, Schwenkglenks M, Meier C (2017) HelsanaArzneimittelreport für die Schweiz 2017. Auswertungsergebnisse der Helsana Arzneimitteldaten aus den Jahren 2013 bis 2016. https://www.helsana.ch/de/helsanagruppe/unternehmen/gesundheitswissenschaften/arzneimittelreport Zugegriffen: 22. Feb. 2018

20. Holt S, Schmiedl S, Thurmann P A (2010) Potentially inappropriate medications in the elderly: the PRISCUS list. Dtsch Arztebl Int 107: 543-51

21. American Geriatrics Society (2015) American Geriatrics Society 2015 Updated Beers Criteria for Potentially Inappropriate Medication Use in Older Adults. J Am Geriatr Soc 63: 2227-46 
22. Häsli T, Bieri G (2013) Die medizinische Versorgung von Patientinnen und Patienten in Schweizer Pflegeheimen. Schweiz Arzteztg 94: 1956-8

23. OECD (2015) Health Data Governance: Privacy, Monitoring and Research. http://dx.doi.org/10.1787/9789264244566-en Zugegriffen: 22. Feb. 2018

24. De Pietro C, Francetic I (2018) E-health in Switzerland: The laborious adoption of the federal law on electronic health records (EHR) and health information exchange (HIE) networks. Health Policy 122: 69-74

25. Schweizerische Konferenz der kantonalen Gesundheitsdirektorinnen und -direktoren (GDK) (2017) Elektronisches Patientendossier - Aktivitäten in den Kantonen. https://www.e-healthsuisse.ch/fileadmin/user upload/Dokumente/2017/D/171219 GDK Notiz Stand Kantone.pdf Zugegriffen: 22. Feb. 2018

26. Bührer A, Kappeler O (2015) eMediplan, der Medikamentenplan unserer Zeit. Schweiz Arzteztg 96: 769-72

27. FMH-Arbeitsgruppe DMA (Direkte Medikamentenabgabe) (2012) Positionspapier zur Ärztlichen Medikamentenabgabe (Selbstdispensation). https://www.fmh.ch/files/pdf9/2012-0227 Positionspapier DMA.pdf Zugegriffen: 22. Feb. 2018

28. Blozik E, Rapold R, Reich O (2015) Prescription of potentially inappropriate medication in older persons in Switzerland: does the dispensing channel make a difference? Risk Manag. Healthc.Policy 8: 73-80

29. Niquille A, Ruggli M, Buchmann M, Jordan D, Bugnon O (2010) The nine-year sustained costcontainment impact of swiss pilot physicians-pharmacists quality circles. Ann Pharmacother 44: $650-7$

30. Ruggli M, Schmutz A, Geiser M, Jörger A (2017) Medikamentenversorgung in Pflegeheimen. https://www.curaviva.ch/files/KON3GUQ/2017 0818 faktenblatt medikamentenversorgung.p df Zugegriffen: 22. Feb. 2018

31. Messerli M, Maes K A, Hersberger K E, Lampert M L (2016) Mapping clinical pharmacy practice in Swiss hospitals: a cross-sectional study. Eur J Hosp Pharm 23: 314-9

32. Bundesamt für Gesundheit (BAG) (2009) Qualitätsstrategie des Bundes. https://www.bag.admin.ch/bag/de/home/themen/versicherungen/krankenversicherung/kranken versicherung-qualitaetssicherung.html Zugegriffen: 22. Feb. 2018

33. Zimmermann C, Fishman L (2017) Der systematische Medikationsabgleich in Schweizer Akutspitälern. Schweizerische Ärztezeitung 98: 1451-3

34. Patientensicherheit Schweiz (2017) Erklärung Sichere Medikation an Schnittstellen. http://www.patientensicherheit.ch/de/themen/Pilotprogramme-progress--/progress--SichereMedikation/Charta-Sichere-Medikation/mainColumnParagraphs/04/download website.pdf Zugegriffen: 22. Feb. 2018

35. Brühwiler L, Niederhauser A, Fishman L (2018) Gefragt ist eine bessere Systematik bei der Überprüfung der Medikation. Curaviva Fachzeitschrift 3

36. Schwappach D L B, Pfeiffer Y, Taxis K (2016) Medication double-checking procedures in clinical practice: a cross-sectional survey of oncology nurses' experiences. BMJ Open 6: e011394

37. Pfeiffer Y, Gut S S, Schwappach D L B (2018) Medication safety in oncology care: mapping checking procedures along the process from prescription to administration of chemotherapy. $J$ Oncol Pract 14: e201

38. Schwappach D L B, Taxis K, Pfeiffer Y (2018) Oncology nurses' beliefs and attitudes towards the double-check of chemotherapy medications: a cross-sectional survey study. BMC Health Serv Res 18: 123

39. Damke B, Stoller R, Leuthold M, Schwappach D L B (2015) Akzidentelle Überdosierungen von Low Dose Methotrexat. Schweizerische Ärztezeitung 96: 1845

40. Ceschi A, Hitz P, Müller L, Kullak-Ublick G, Piffaretti V (2018) Aktive elektronische Überwachung der unerwünschten Arzneimittelwirkungen bei hospitalisierten Patienten. https://www.fmh.ch/files/pdf20/Elektron. Ueberwachung unerwuenschter Arzneimittelwirkung en Gewinner Innovation Qualite 2018.pdf Zugegriffen: 5. Jun. 2018

41. Niederhauser A, Zimmermann C, Fishman L, Schwappach D (2018) The implications of involving pharmacy technicians in obtaining a best possible medication history from the perspectives of pharmaceutical, medical and nursing staff: a qualitative study. BMJ Open 8: e020566 
42. Hersberger K E, Messerli M (2016) Development of Clinical Pharmacy in Switzerland: Involvement of Community Pharmacists in Care for Older Patients. Drugs Aging 33: 205-11

43. Costa F A, Scullin C, Al-Taani G, et al. (2017) Provision of pharmaceutical care by community pharmacists across Europe: Is it developing and spreading? J Eval Clin Pract 23: 1336-47

44. Messerli M, Blozik E, Vriends N, Hersberger K E (2016) Impact of a community pharmacist-led medication review on medicines use in patients on polypharmacy - a prospective randomised controlled trial. BMC Health Serv Res 16: 145-61

\section{Legende für die Abbildung}

Abbildung 1: Personelle Ressourcen für klinisch-pharmazeutische Leistungen (Rote Balken = Summierte Vollzeitäquivalente in 33 Krankenhäusern mit klinischer Pharmazie) in Regionen ohne Selbstdispensation (dunkelgrün), Mischform (grün) sowie mit Selbstdispensation (hellgrün). Rote Kästchen ohne Füllung = Kantone ohne jegliche klinisch-pharmazeutische Ressourcen. (Quelle: Dr. Markus Messerli, Universität Basel)

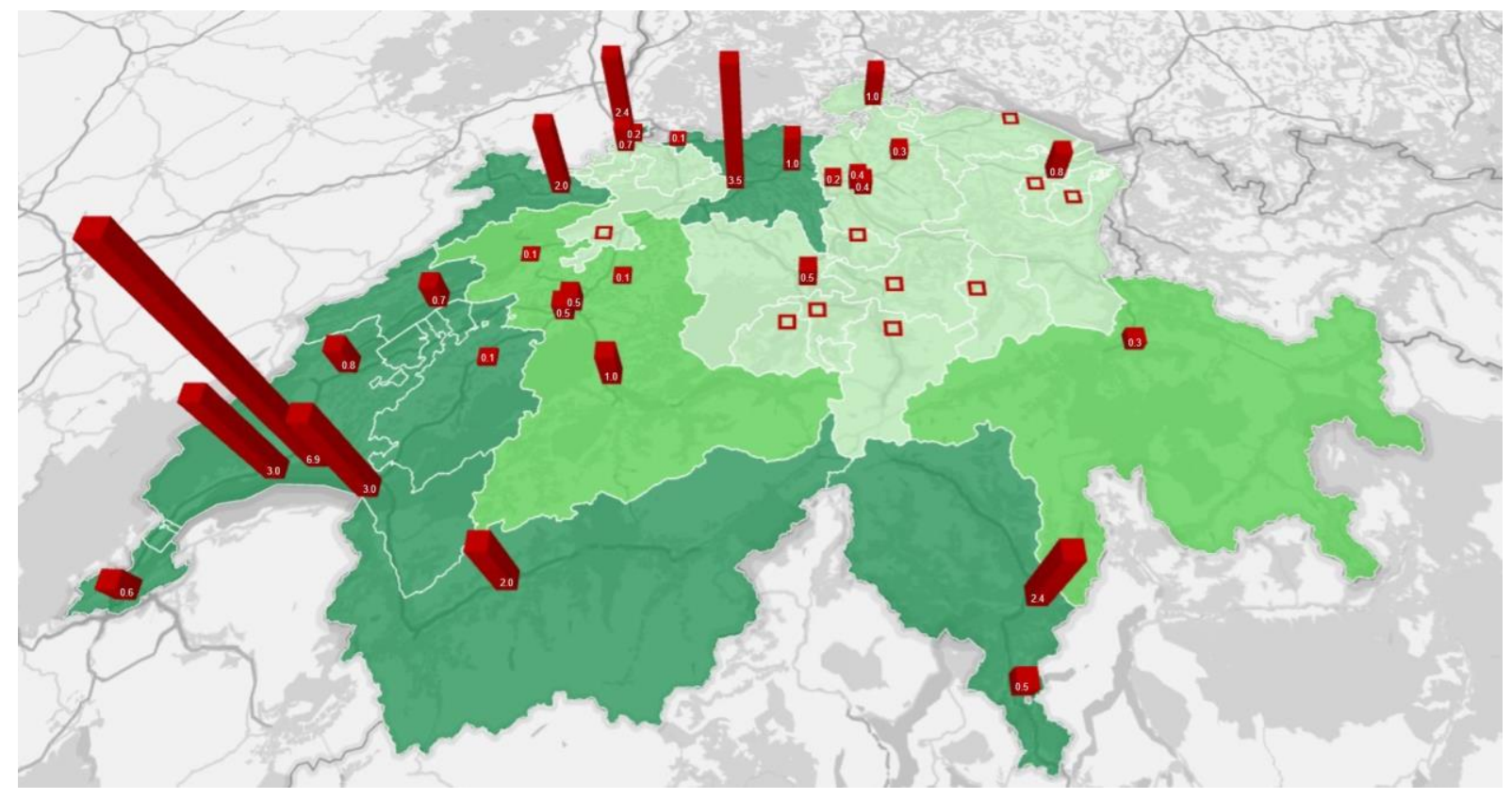

